\title{
Enabling a 'Celalettin-Field Quantum Observation Tunnel' to Develop a Hand Held Medical Resonance Imaging Device in order to prevent Paediatric Sedation during Medical Imaging - A Short Communication
}

\section{Metin Celalettin*, Hasret Celalettin and Horace King}

Victoria University, Institute of Telecommunications, Electronics, Photonics and Sensors, Melbourne, Australia

*Corresponding Author: Metin Celalettin, Victoria University, Institute of Telecommunications, Electronics, Photonics and Sensors, Melbourne, Australia.

Received: August 21, 2019; Published: August 30, 2019

\begin{abstract}
The 'Celalettin-Field Quantum Observation Tunnel's (CFQOT) sole purpose is to destroy a quantum system's ability to act quantum mechanically. Specifically, the CFQOT causes a quantum entangled system to achieve quantum decoherence via 'quantum observation'. The CFQOT was originally designed as a means of quantum observation to inhibit the function of a near-future military quantum radar in order to protect the stealthyness of fighter jets. However due to the similarities between how it is purported to function and a Medical Resonance Imaging (MRI) device, it is proposed that MRI-like imaging could be instilled in a hand held device for paediatric use using a longer wavelength. This is enabled by the chemical engineered particle reservoir used simultaneously to the CFQOT in military studies.
\end{abstract}

Keywords: Medical Resonance Imaging (MRI); Celalettin-Field Quantum Observation Tunnels (CFQOT); Imaging

\section{Introduction}

A chemical engineered particle reservoir could be exploited in an incapsulated hand held device and applied to the surface of paediatric patient's skin in order to produce an image via quantum entanglement based remote sensing, avoiding the need for sedation in many instances [1]. This is enabled by a spin exchange optical pumping capability with applied anti-ferromagnetism on the electrons in the molecular make-up of the patient's tissue rather than the protons $[3,4]$. Electrons are much smaller and therefore require a lower and thereby safer wavelength, and spin polarization would occur using an electromagnet attached to the device [1]. Quantum entangled particles within the device's chamber would produce the image, which is the crux of the technology however the rate of quantum computing advancements have a direct impact on the timeliness of such a device's development.

Gaps in quantum theory research preventing nuclear imaging advancements

Current research into Quantum Theory is heavily focussed on overcoming the quantum decoherence problem, predominately in the race to build a functioning quantum computer [2]. Current research into quantum decoherence is focussed predominately on how to overcome it, rather than how to exploit it. Quantum computers are going to change the world; the computing power that we see in supercomputers will be logarithmically overtaken in terms of performance, and even research will progress at an incredibly overwhelming rate $[2,3]$.

\section{Extant MRI machines}

Extant MRI machines focus on hydrogen atoms in human tissue due to the abundance of water and fat in the body. An electric field is applied in pulses which polarizes protons within these hydrogen atoms. Different imaging contrasts are generated via signals sent to a processor based on the amount of time it takes the polarized nuclear spins of these protons to return to their angular momentum and low energy state at each pulse [3,4]. Differences in contrasts are due to the density of hydrogen atoms dispersed throughout the body, the molecular structure of different tissues in the body, and their associated bonds [5].

'Spinstronics' is the discipline of spin polarizing electrons [6]. The reason why current models are so large is because they need to produce a constant rate and strength of magnetic fields. The reason the patient needs to be inserted into the machine is for those mag- 


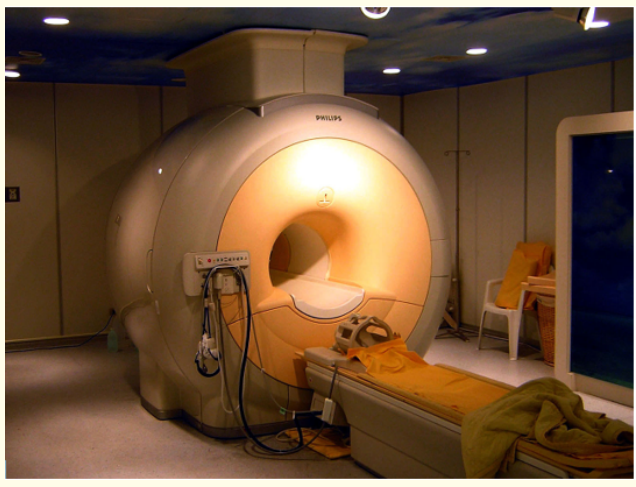

Figure1: Extant MRI machines.

netic fields to capture the amount of time it takes for the nuclear spins of the protons within the hydrogen atoms by direct exposure [3-5], rather than the proposed remote sensing in this study.

At Figure 2, the 'Celalettin-Field Quantum Observation Tunnel' (CFQOT) is a theoretical speculative structure produced in an ensemble of Orbital Angular Momentum spin polarized particles with an applied anti-ferromagnetism; a magnetization that exhibits the magnetic moments of the atomic ensemble [1]. The mathematical proof of concept enables what would be required to develop a hand held MRI, based on the same quantum entangled based technology $[1,2]$.

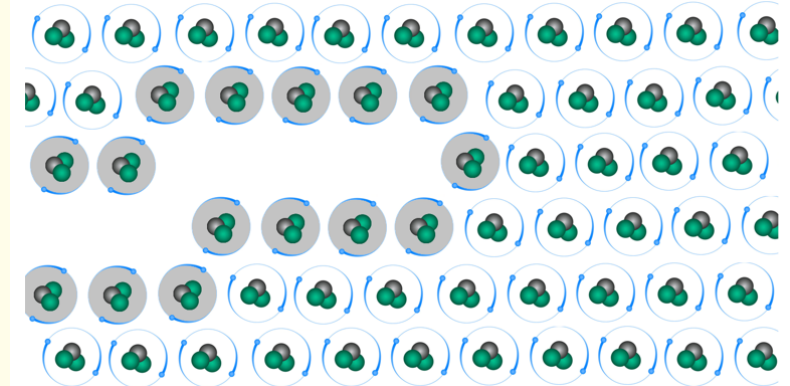

Figure 2: 'Celalettin-Field Quantum Observation Tunnel'. (Helium-3 atoms in grey have been depolarized).

Given the CFQOT, mathematically described by:

$$
\begin{aligned}
& \nabla^{2} \psi+\frac{8 \pi^{2} m}{h^{2}}(E-V) \psi=0 \\
& +\frac{d^{2} y}{d x^{2}}+[a-2 q \cos (2 x)] \mid y=0 \\
& \frac{\exists \mathcal{L}_{\text {SQED }} \exists X}{t}
\end{aligned}
$$

\begin{tabular}{|c|c|c|}
\hline The $\gamma_{\text {sig }}$ as the wave cquation & 'existing with $(\exists)$ ' & $\nabla^{2} \Psi^{\prime}+\frac{8 \pi^{2} m}{h^{2}}(E-V)^{\Psi} \Psi^{\prime}=0$ \\
\hline Mathieu differential equation & 'existing with $(\exists)^{\prime}$ & $\frac{d^{2} y}{d x^{2}}+[a-2 q \cos (2 x)] y=0$ \\
\hline Lagrangian plasma QED equation & 'existing with $(\exists)$ ' & $L_{S E D}$ \\
\hline $\begin{array}{l}\text { Lorentz quantum parametcr causing OAM } \\
\text { polarization (Spin-Spin Coupling) }\end{array}$ & 'existing with $(\exists)$ ' & $x$ \\
\hline $\begin{array}{l}\text { Time it takes the electromagnet to re-polarize } \\
\text { the depolarized atoms }\end{array}$ & 'over' & $\mathrm{t}$ \\
\hline
\end{tabular}

Given the enabling equations for the CFQOT formulation [1]:

In future articles, the mathematical proof of concept will be used to derive the appropriate parameters required for paediatric use as regulated. See impression of the proposed quantum entanglement technology based remote hand held sensing MRI, as proposed in Figure 3.

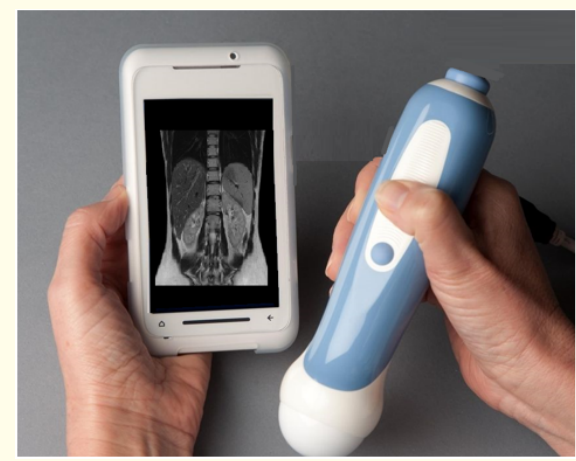

Figure 3: Proposed hand held MRI for paediatric patients.

\section{Conclusion}

A short communication considering an initial proposal for a hand held MRI machine for paediatric patients is described. A mathematical proof of concept for the CFQOT is provided which was initially designed to protect fighter jets from a military quantum radar. Almost identical quantum attributes enabling the CFQOT and its corresponding chemically engineered particle reservoir in current literature provide for a hand held MRI machine to be developed. Such a device would be of an enormous benefit in medicine where the need to sedate paediatric patients to image them, and will be expanded upon in an article in the near future.

\section{Bibliography}

1. Celalettin M and King H. "The 'Celalettin-Field Quantum Observation Tunnel'; a Quantum Communication Countermeasure Speculative Structure". Scholar Journal of Applied Sciences and Research 1 (2018): 5-9.

2. Gisin N and Thew RT. "Quantum communication technology". Electronics letters 46 (2010): 965-967. 
3. Kane BE. "A silicon-based nuclear spin quantum computer". Nature 393 (1998): 133.

4. Feinberg DA., et al. "Multiplexed echo planar imaging for subsecond whole brain FMRI and fast diffusion imaging". PLOS One 5 (2010): e15710.

5. Laniado M., et al. "First use of GdDTPA/dimeglumine in man". Physiological Chemistry and Physics and Medical NMR 16 (1984): 157-165.

6. Wolf SA., et al. "Spintronics-A retrospective and perspective". IBM Journal of Research and Development 50 (2016): 101-110.

Volume 2 Issue 9 September 2019

(C) All rights are reserved by Metin Celalettin., et al. 